DOI: https://doi.org/10.46630/phm.12.2020.11

Snežana U. Gudurić ${ }^{1}$

Article de recherche

Université de Novi Sad

УДК $811.163 .41^{\prime} 373.45=133.1(497.113)$

Faculté de Philosophie ${ }^{2}$

$323.15(=133.1)(497.113)$

Département d'études romanes

Reçu : le 1/3/2020

\title{
À LA TRACE DES FRANÇAIS DE VOÏVODINE
}

La Voïvodine, province septentrionale de la Serbie, a toujours été une des mosaïques les plus variées de nations, langues et cultures en Europe. Dans cet article, notre intérêt s'est porté principalement sur les traces des migrations de la population française, organisées par la monarchie austro-hongroise au cours du XVIII ${ }^{\mathrm{e}}$ siècle. À cette époque-là, un nombre important de Français est venu s'installer en Voïvodine, plus particulièrement au Banat, l'une des trois régions de ladite province. La présence de cette population, de sa langue et de sa culture, n'est plus évidente, mais une recherche historico-linguistique, dévoilera dans certains noms de famille voïvodiniens, ainsi que dans quelques micro-toponymes du Banat, les traces cachées des Français d'autrefois. Les formes premières des noms de famille et des toponymes ont subi des modifications phonético-phonologiques importantes dues respectivement à la germanisation, la magyarisation et à la serbisation, on les a découvertes grâce à une recherche minutieuse dans les archives suivie d'une analyse linguistique. Dans cette communication, nous présenterons les résultats des recherches interdisciplinaires, historiques et linguistiques, présentant les racines françaises de nos voisins, dont les traces ne se sont pas complètement perdues tout au long des époques passées, grâce, avant tout, aux travaux de Nicolas Hess, Felix Milleker, Franz Stanglica, André Rosambert et plus particulièrement de ceux de Milorad Arsenijević.

Mots clés : onomastique, noms de famille, toponymes, Voïvodine, colonisations, Français, Milorad Arsenijević

\section{Introduction}

La Voïvodine, province limitrophe au nord de la Serbie, entre la Roumanie, la Hongrie et la Croatie, composée de trois régions (Syrmie, Banat et Batchka), ne cesse de passionner, depuis le XVIII ${ }^{e}$ siècle, par sa

\footnotetext{
${ }^{1}$ snezana.guduric@ff.uns.ac.rs

${ }^{2}$ Cet article est rédigé dans le cadre du projet $\mathrm{N}^{\circ} 178002$ Langues et cultures dans le temps et dans l'espace, soutenu par le Ministère de l'éducation, du développement technologique et de la science de Serbie.
} 
mosaïque extraordinaire de populations, langues et cultures. Les migrations, les colonisations et les guerres ont créé une des régions les plus mélangées en Europe d'aujourd'hui. L'histoire, surtout les événements datant du XVIII ${ }^{\circ}$ siècle, a laissé des traces émouvantes dans tous les segments de la vie de ses habitants. Ces traces sont particulièrement visibles dans les toponymes et dans les patronymes des Voïvodiniens dont la plupart de par leur forme indiquent plus ou moins explicitement l'origine ethnique de ceux qui les portent.

Les enquêtes liées à l'installation des colons dans cette région ont principalement été menées par des historiens et des ethnographes, parmi lesquels nous citons ceux qui ont porté un intérêt plus particulier aux colons d'origine française : Felix (Srećko) Milleker (en 1886, 1878, 1933, 1937), Frantz Stanglica (1935), Nicolas Hess (1928, 1939 et 1940), André Rosambert (1952), Milan Petrov (1974), Jean-Marc Becker (1990), Emile Botiş (1946). En ce qui concerne les recherches linguistiques, les contributions les plus importantes ont été apportées par Georges Rebattet ${ }^{3}$ (dans les années 60 du $X^{\mathrm{e}}$ siècle) et Milorad Arsenijević $(1990,1992,1996)$. Au début du troisième millénaire, ce sujet à suscité l'intérêt de nouveaux chercheurs en linguistique, entre autres, Snežana Gudurić $(2007,2010,2013,2017)$ et Simona Goicu (Goicu-Cealmof) (2000, 2007).

Les études et articles publiés entre 1886 et 2017 offrent un nombre important de données sur le voyage, l'installation et la vie des colons, mais aussi sur les difficultés qu'ils ont rencontrées au cours des siècles qui ont suivi leur arrivée. Les recherches linguistiques portent plus particulièrement sur les changements de formes des toponymes et des patronymes, étant donné que ces formes, d'une époque à l'autre, s'adaptaient aux langues différentes selon des conditions spécifiques historico-politiques.

\section{Cadre historique}

Au XVIII ${ }^{\mathrm{e}}$ siècle, l'Autriche, s'emparant définitivement du territoire du Banat et de la Batchka, au nord du Danube, entama de nombreux projets, dont les plus importants portaient sur la fortification des nouvelles frontières, sur le renforcement économique de l'Empire et sur la modification du profil démographique des pays devenus allemands, ainsi que de ceux qui devaient le devenir. À cet effet, l'état impérial avait adopté une organisation administrative particulière pour les régions en question : la Batchka avait obtenu un statut spécial et le Banat était devenu domaine impérial. Le premier administrateur

\footnotetext{
${ }^{3}$ Attaché pour la culture auprès de l'Ambassade de France en Yougoslavie, ses listes des prénoms et des noms de famille d'origines françaises rassemblés en Voïvodine lors de son service en Yougoslavie, n'ont jamais été publiées.
} 
des pays nouvellement acquis, le général-commandant Claudius Florimund Mercy, originaire de Lorraine, était responsable pour la réalisation des objectifs cités ci-dessus ${ }^{4}$.

Comme la population en Europe était épuisée par la pauvreté, des guerres et des corvées publiques, on a fait appel avant tout à des Allemands, puis à des Luxembourgeois, à des Lorrains ainsi qu'à d'autres populations vivant en Empire ottoman (plus particulièrement aux Serbes et aux Roumains)

Afin d'assurer la nouvelle répartition démographique des territoires en question, « l'État impérial organisa trois colonisations à l'aide desquelles on visait à faire venir avant tout des Allemands pour renforcer le pouvoir défensif du pays, augmenter le nombre de contribuables et cultiver les terres » (ARSENIJEVIĆ 1992 : 18). À cette époque-là, l'Allemagne était surpeuplée et, par ailleurs, les Autrichiens n'étaient pas du tout visés à devenir des futurs citoyens de nouvelles terres, étant donné qu'on ne voulait pas dépeupler le pays central.

La première colonisation s'effectua après la retraite des Ottomans et la paix de Požarevac en 1718 jusqu'à la période marquée par la fin de la guerre austro-turque, 1736 et 1739. C'était l'époque où, après 154 ans de domination turque, le Banat passa sous l'administration austro-hongroise. Les colons venaient de Trèves, Cologne, Mayence, Souabe, Lorraine, du Palatinat, Wurtemberg, Luxemboug, de l'Alsace, mais aussi de l'Espagne - ajoutons que ces derniers passaient par Wienne. Il y avait aussi un certain nombre d'Italiens, qui étaient liés par des liens de parenté avec des Espagnols ${ }^{5}$, des Tchèques, des Slovaques et des Magyars aussi. Il y avait parmi eux des employés, des artisans, des forestiers, des mineurs et des agriculteurs.

Tout colon obtenait un passeport particulier, ses frais de voyages étaient payés par l'État et chaque personne âgée de plus de 15 ans avait le droit à un talir (un florin et 30 couronnes). Le lieu de rassemblement était Ulm, sur le Danube, d'où les colons, en passant par Vienne, étaient dirigés vers la Batchka et le Banat où les attendait la Commission colonisatrice (STANGLICA 1937 : 44). Des Lorrains, auxquels nous portons un intérêt particulier dans cet article, furent installés à Mercydorf et à Bruckenau ${ }^{6}$. Chaque famille obtenait une maison avec au moins 2 pièces et une cuisine, une étable, du bétail, des instruments aratoires, du bois pour le chauffage ainsi que 24 arpents de labour, 6 arpents de prairie, un arpent de jardin et de la semence. Pour faciliter la vie

\footnotetext{
${ }^{4}$ Il s'agissait avant tout de développer l'agriculture, la viticulture et les métiers, ainsi que d'assurer le travail dans les mines, la construction du canal de Béga, le transport et le dessèchement des marécages.

${ }^{5}$ À cette époque-là, l'Autriche avait perdu Naples et Sicile et les fonctionnaires d'origine espagnole ainsi que les Italiens loyaux aux Autrichiens devaient quitter le pays.

${ }^{6}$ Aujourd'hui Carani et Pişchia en Roumanie.
} 
des nouveaux arrivés, l'État les dispensa de 3/4 des impôts pendant 3 ans, puis de 2/4 - ils ne seraient dans l'obligation de payer le total des impôts qu'après 12 ans - ainsi que de la mobilisation et de la corvée publique. De plus, ils pouvaient se procurer des pierres, produire de la chaux, ramasser de l'argile et faire des briques, produire et vendre de la bière, construire des moulins. Ils étaient installés pour la plupart dans des villages construits selon des plans rigoureux, avec une rue, semblables à ceux en Lorraine. L'infrastructure de chaque village était assurée par des ingénieurs militaires de l'État, à savoir la route, l'église, le presbytère, le moulin, la fontaine et l'école avec les maîtres payés par l'État (STANGLICA 1935 : 16, BECKER $1990: 3$ ).

La guerre de 1736, la peste et les autres maladies dues aux terrains marécageux, ravagèrent la nouvelle terre qui se vit de nouveau dépeuplée.

Les autorités impériales recoururent encore une fois à une colonisation organisée. Celle-ci eut lieu entre 1745-1772 et fut la plus forte. Le pouvoir impérial faisait même da la publicité dans la presse en Lorraine et en Bourgogne, et partiellement en Franche-Compté, voulant dépeupler ces régions de la population française (STANGLICA 1935 : 30). La plupart des colons s'orientaient vers la Batchka et le Nord-Est du Banat, et surtout jusqu'aux environs de Timisoara. L'Autriche fit venir avant tout des paysans de Lorraine, puis quelques ouvriers, artisans et lainiers, ainsi que des viticulteurs de Bourgogne. Il y avait aussi des particuliers qui faisaient venir des groupes de colons pour s'assurer une maind'œuvre ${ }^{7}$. Des Lorrains furent particulièrement nombreux à quitter leur patrie étant donné la vie extrêmement difficile dans leur pays : les impôts élevés, les inondations, les pénuries alimentaires, le coût de vie élevé, et surtout le recrutement et la mobilisation au cours de la guerre de sept ans.

Les autorités françaises tentèrent d'empêcher l'émigration, mais les ordonnances des ducs de Lorraine furent pratiquement sans succès de même que les procédures judiciaires dans le baillage de Bouzonville (Archives Nationales : État général des fonds, Série K - Cartons in ARSENIJEVIĆ 1992 : 25, GOICU-CEALMOF 2007 : 117). C'est pourquoi la Cour Souveraine de Lorraine émit un arrêté, le $1^{\text {er }}$ juin 1769 , permettant les arrestations à la frontière, l'annulation des contrats de vente établis dans un but d'émigration et d'autres sanctions contre les officiers locaux qui n'arrêtaient pas les émigrants (BECKER 1990 : 4, ARSENIJEVIĆ 1992 : 24-25). Pourtant, toutes ces mesures ne suffirent pas à arrêter l'émigration.

Entre 1752 et 1754, environ 2000 Lorrains vinrent au Banat et s'installèrent à Mercydorf ${ }^{8}$, attendant de bénéficier de la fin de la construction

\footnotetext{
${ }^{7}$ Par exemple, un certain Johan Oswad réussit à faire venir au Banat environ 1600 personnes de Lorraine, de Trèves, de Mayence et de Luxembourg (ARSENIJEVIĆ 1992 : 21).

${ }^{8}$ D'après dr Schiff, médecin de Mercydorf, dans ce village il y avait $18 \%$ d'Allemands, $58 \%$ de Français et 24\% d'Italiens et d'Espagnols (ROSAMBERT 1952 : 53).
} 
de nouveaux villages. Une nouvelle vague amena encore 12065 colons lorrains entre 1763 et 1774 .

La troisième colonisation s'effectua entre 1783 et 1787 et se distingua par un nombre considérable d'Italiens, surtout des éleveurs de vers de soie et des cultiveurs de riz (PETROV 1974), tandis que le nombre de Français, s'installant cette fois dans des communautés mixtes franco-allemande (Novi Kozarci et Bogoros en Serbie, puis Gottlob, Sankt Nikolaus Gross, Lowrin Tomnatic/Osterne en Roumanie) ne fut que symbolique (STANGLICA 1935, ARSENIJEVIĆ 1992 : 39, GOICU 2000 : 177).

La plupart des colons français arrivés lors des trois colonisations, furent stationnés au Banat, appartenant aujourd'hui à la Roumanie. Pour ceux qui s'installèrent en Voïvodine actuelle, le pouvoir austro-hongrois bâtit trois villages - Charleville, Saint Hubert et Seultour. (HESS 1927).

Après la Grande révolution française un certain nombre d'émigrés français, après des années d'errance en Europe, se sont arrêtés au Banat, où ils ont été accueillis par des familles françaises. La plupart d'entre eux sont rentrés en France après l'adoption de la Constitution civile par le clergé (liberté du service religieux (1795). Un certain nombre de vieux colons souhaitèrent retourner dans leur pays d'origine, mais les autorités leur ont demandé de rembourser tous les fonds investis par l'État.

Très peu de rapatriés de ce type ont été enregistrés. (HESS 1927 : 198, ROSAMBERT 1952 : 56, ARSENIJEVIĆ $1992: 34$ )

D'après le recensement fait en 1770, il y avait 42201 colons français, en 1840 ils n'étaient que 6150 , tandis que les résultats du recensement de 1900 ne distinguaient plus du tout cette nationalité, même pas dans la catégorie autre qui comprenait les Tziganes, les Bulgares, les Shocatzes et les Krassovans (BOTIŞ 1946 : 17-18, GOICU-CEALMOF 2007 : 117).

\section{3. Šarlevil, Sveti Hubert et Soltur}

Comme nous l'avons mentionné plus haut, entre 1769 et 1772 le pouvoir austro-hongois construisit trois villages pour les colons français - Charleville, Saint-Hubert et Seultour. Les deux premiers furent nommés d'après les villages autour de Metz ${ }^{9}$ d'où venaient la plupart des colons. Le troisième obtint son nom d'après une tour solitaire autour de laquelle la nouvelle colonie avait été érigée. Jusqu'à août 1772, 80 maisons furent construites à SaintHubert et 60 à Charleville et à Seultour ${ }^{10}$. Chaque village fut gouverné par un

\footnotetext{
${ }^{9}$ Les colons installés dans ces trois villages venaient tous de Lorraine, notamment de Metz, d'Arracourt, de Francheville, de Moyenvic, de Dorsweiler, de Bergaville et de Dieuze).

${ }^{10}$ Toutes les données historiques concernant ces trois villages sont reprises de Hess 1928 et
} 
ancien résident local qui se trouvait directement sous 1'autorité de Timişoara, contrairement aux villages allemands sous l'autorité locale de Kikinda. À partir de septembre 1772, les registres de naissances, mariages et décès, pour tous les trois villages, furent tenus à Saint-Hubert, où se trouvait aussi le cimetière local. Le service religieux fut d'abord organisé dans une petite église en bois, remplacée quelques années plus tard par un édifice en matériel solide, mais sans tour.

L'école commença à fonctionner dès 1771 et fut obligatoire pour les enfants de 9 à 15 ans, mais uniquement dans la période entre décembre et mars, étant donné que du printemps jusqu'à l'automne les enfants aidaient leurs parents dans les champs. En 1781, il y avait 63 enfants à l'école de SaintHubert, 39 à Charleville, et 38 à Seultour. Au cours des années suivantes, l'allemand supplanta petit à petit le français, jusqu'à la proclamation du 6 décembre 1777, par laquelle la langue française fut définitivement éliminée du système scolaire du Banat pour céder la place à l'allemand ou au latin. Pourtant, le français persista à l'Église, et dans les registres paroissiaux d'état civil où l'on enregistrait encore des prénoms sous la forme latinisée et le nom de famille en graphie française (Ludovica Canton, Ioannes Baptista Lamoulin, Nicolaus La Fleur, etc.).

La nouvelle administration modifia très vite le nom de Seultour d'abord en Soltour, puis en Soltur, en adaptant sa graphie à la prononciation allemande.

En 1867, d'après un accord austro-hongrois, la Hongrie prit en charge le gouvernement de la région du Banat ${ }^{11}$ et imposa le hongrois comme langue administrative. Très vite, dans les documents officiels Charleville devint Károlyliget et Soltur Szentborbála, tandis que Saint-Hubert garda son nom, les administrés hongrois voulant respecter le nom du saint.

Cette situation dura jusqu'à la fin de la Grande guerre, après laquelle une partie du Banat ${ }^{12}$, avec les trois villages anciennement français, passa sous l'administration du Royaume de Serbie. Au cours d'un siècle et demi, les trois agglomérations, agrandies et peuplées d'autres nations, pour la plupart des Serbes, se sont fusionnées en une seule, nommée Veliko Banatsko Selo (Le Grand Village du Banat) dont les quartiers portent aujourd'hui les noms des anciens village français - Šarlevil, Soltur et Sveti Hubert, alors que seul le premier laisse encore deviner ses origines françaises.

\footnotetext{
Arsenijević 1992.

${ }^{11}$ Ainsi que des régions de la Batchka et de la Syrmie, constituant aujourd'hui des parties de la Voïvodine.

${ }^{12}$ Après la Grande guerre le Banat est partagé entre la Serbie, la Hongrie et la Roumanie.
} 


\section{Les noms de famille français en Voïvodine}

La troisième génération des Français, sous la pression intense de leurs voisins, d'abord allemands et ensuite hongrois, a perdu ses coutumes et sa langue et s'est complètement noyée dans les ethnies voisines (allemande, hongroise, rarement serbe, à cause de l'incompatibilité religieuse) et à la fin $\mathrm{du} \mathrm{XIX}^{\mathrm{e}}$ siècle seules quelques vieilles femmes priaient encore en français ${ }^{13}$. Pourtant, au début du $\mathrm{XX}^{\mathrm{e}}$ siècle, les voyageurs qui sont passés par le Banat notaient :

« Certains habitants parlent un patois souabe imprégné de mots et de structures français et ils portent des noms, toujours latins, qui rappellent leur pays d'origine » (HESS 1927, SASSU 1928 in GOICU-CEALMOF 2007, STANGLICA 1935).

Pourtant, la plupart des noms de famille d'origine française, de par leur forme, cachent aujourd'hui leur origine. Il est bien difficile, par exemple, de reconnaître les formes françaises Sébastien, July et Charon dans celles de Šebešćan, Silić et Šaronjev. Il y en a tout de même, bien que très rares, qui laissent deviner leur forme originale - Berar (Bérard) ou Šambor (Chambord).

Tous les noms de famille français ne se sont pas modifiés simultanément et de façon unique. Un certain nombre de noms des colons avaient déjà subi des transformations sur les listes de voyage, établies à Ulm et à Vienne. Cette première adaptation orthographique, faite par des administrés colonisateur chargés de noter toute famille et personne migrante, témoigne de l'influence de l'orthographe et de la prononciation de leur langue maternelle - l'allemand. Ainsi, on notait Michael ou Mischel (le même nom était noté différemment sur deux listes) Dukarn au lieu de Michel Ducarne, Wilar au lieu de Villard, Johann Baptist Kaschno au lieu de Jean-Baptist Cachenot, Matje au lieu de Mathieu, Lefor au lieu de Lefort, Lakaj au lieu de Lacaille, Majet au lieu de Mayette, Rischar au lieu de Richard.

Comme les administrés allemands n'étaient pas très sensibles à la distinction des phonèmes voisés et non-voisés, ils confondaient des consonnes sonores et sourdes et notaient Bikar au lieu de Picard, Teschan au lieu de Dejean, Barmantje au lieu de Parmentier, Bless au lieu de Blaise, Kotre au lieu de Cauderet, Lutje au lieu de Loudier ${ }^{14}$.

Les voyelles nasales présentaient une dificulté de plus, pratiquement insurmontable pour les administrés colonisateurs. Comme ce type de voyelles

\footnotetext{
${ }^{13}$ Hess note que, dans cette région, les dernières personnes parlant entre elles en français étaient Anna Muschong, née Kostett à Saint-Hubert, Barbara Lettang, née Pier, et Charlotte Martin, née Letang, à Charleville, et Peter Hanrio de Seultour, mort en 1866 avec lequel le français est définitivement mort en tant que langue maternelle de cette population. (HESS $1928: 115$ )

${ }^{14}$ La liste des noms est prise de Arsenijević 1992 : 76-86, qui s'appuie principalement sur Hess 1928 .
} 
n'existe pas en allemand, ils orthographiaient les noms des colons selon leur propre impression auditive, qui n'était même pas toujours identique chez un même administré. En général, les uns ne faisaient que supprimer la voyelle nasale en ajoutant un $-n$ final, les autres, percevant une consonne vélaire à la fin du nom, la notaient conséquement. En fin de compte, on trouve sur des listes de colons des formes telle que : Loran pour Laurent, Teschan pour Dejean, Leblan pour Leblanc et Kohlen pour Colin. Sur une autre liste, Martin est devenu Marteng, Perrin $\rightarrow$ Perreng, Colin $\rightarrow$ Koleng, Vautrin $\rightarrow$ Wottreng, Mougeon $\rightarrow$ Muschong, Lapin $\rightarrow$ Laping, Marchand $\rightarrow$ Marschang, Amant $\rightarrow$ Hammang.

Les deux différents noms de famille, Masson et Maçon étaient orthographiés de la même façon - Massong, tandis que Dupont apparaissaient en trois formes distinctes - comme Dippong, Diebong et Düppong.

Il y avait aussi de fausses orthographes dues aux images acoustiques de lexèmes semblables en français et en allemand. Ainsi, Blême est devenu Blum ('fleur'), Leblanc $\rightarrow$ Liebling ('aimé') et Vielhomme $\rightarrow$ Willkomm ('bienvenu').

Les quelques cas de traductions sont plus tardifs et se résument pratiquement à Renard devenu Fuchs et Lenoir devenu Schwarz.

Dans les archives (au début c'étaient des listes de colons, plus tard, il s'agissait plutôt de documents judiciaires) on trouve plusieurs formes d'un même nom de famille : Thibault apparaît sous les formes Dippold, Dibold, Diebold, Tibolt, Düpold et Thiebo ; Aubertin est noté comme Oberting, Oberteng, Oberding et Obertin ; Lenard comme Lenart, Lenhar, Lenhard et Lenhart.

En 1867, un accord austro-hongrois ouvre la porte à une implantation massive de l'élément national magyar dans la région du Banat et par la suite, à une forte magyarisation.

Les noms des descendants des colons français ont subi une nouvelle transformation, qui, cette fois, touchait surtout les prénoms : Paul est devenu Pál, Nicolas $\rightarrow$ Miklos, Pierre / Peter ${ }^{15} \rightarrow$ Péter, François / Franz $\rightarrow$ Ferenz, Jean / Johan(n) $\rightarrow$ János, Michel / Michael $\rightarrow$ Mihály, Georges / Georg $\rightarrow$ György, Louis $\rightarrow$ Lájos.

Un certain nombre de noms de famille ont aussi été magyarisés : on orthographiait Gaudenyi pour Gaudin, et Martegnyi pour Martin et Marting.

La fin de la Grande guerre apporta, avec la nouvelle carte de l'Europe désignant la Voïvodine comme une région du Royaume de Serbie, une nouvelle, quoique sporadique, -isation. Vu que l'écriture serbe était cyrillique et que les noms de famille serbes présentaient des terminaisons particulières,

${ }^{15} \mathrm{La}$ double forme indique les cas de germanisation des prénoms français. Que le prénom soit en forme française ou allemande, il finit par se magyariser. 
certains noms initialement français, puis germanisés, ensuite magyarisés ont fini par être serbisés, et ce par l'ajout des suffixes $-o v,-e v$ et -ić. C'est ainsi qu'aujourd'hui, on trouve en Voïvodine des noms de famille tels que Aras, Araskov et Araškov; Sebastian, Sebastijan, Šebě̌čen, Šebě̌ćen et Šebešćan ; Šaru, Šaro et Šaronjev, Berar et Berarov ; Lenard, Lenart et Lenartić, Solar et Solarov ; Soldat, Soldatić et Soldatović; Šili et Šilić ; Žak, Žakanov et Žakonov etc., qui impliquent souvent l'ethnie et la confession de ceux qui les portent.

\section{En guise de conclusion}

Bien qu'installée dans les trois villages strictement français, la population française en Voïvodine n'a pas réussi à conserver sa langue et ses coutumes. Au cours d'un siècle et demi, elle s'est complètement noyée dans les ethnies voisines, d'abord allemande, puis hongroise et, pour de rares cas, serbe $^{16}$ (à cause de l'incompatibilité religieuse), ne nous laissant que quelques traces plus ou moins cachées de sa présence sur ces terrains. Ces traces se résument avant tout à environ 200 noms de famille, transformés d'abord par la germanisation, puis par la magyarisation et partiellement par la serbisation, mais aussi aux trois microtoponymes, désignant les trois anciennes colonies françaises fusionnées à présent dans l'agglomération de Veliko Banatsko Selo (Le Grand Village du Banat). Des traces plus nombreuses se trouvent aujourd'hui en Roumanie, étant donné que la majorité des colons français s'étaient installés dans le Banat roumain.

Nous nous devons de mentionner ici plus particulièrement le nom de Milorad Arsenijević, professeur de linguistique romane à l'Université de Novi Sad, dont la thèse de doctorat, intitulée Les noms de famille italiens et romans occidentaux, soutenue en 1992 à la Faculté de Philosophie de Novi Sad, a servie de base pour cet article. Il s'agit de l'étude linguistique la plus complète des patronymes des Voïvodiniens d'origine romane, mais qui n'est toujours pas publiée. Ayant fait partie du grand projet européen PATROM sur les patronymes romans en Europe, cette étude mérite d'être plus visible et plus accessible non seulement aux chercheurs mais aussi à tous ceux qui s'intéressent à l'histoire, aux noms de famille, aux origines des uns et des autres, voisins plus ou moins proches, et surtout à la mosaïque extraordinaire des populations de la Voïvodine.

APPENDIX. Liste des noms de famille d'origine française qu'on trouve

\footnotetext{
${ }^{16}$ Les descendants des colons français se déclarent aujourd'hui pour la plupart Hongrois, puis Croate, ensuite Serbe. Le moins nombreux sont ceux qui se déclarent Allemands.
} 
aujourd'hui en Voïvodine ${ }^{17}$ :

Andre, Aras, Araskov, Araškov, Azlen, Balej, Balaj, Berar, Berarov, Bikar, Bles, Bobek, Bodre, Bokroš, Bomble, Bone, Bonert, Bosak, Bošan, Botan, Bovan, Brumer, Brunet, Čamber, Čambor, Šambor, Čief, Daku, Debre, Devald, Deman, Demendi, Dipold, Donat, Dupor, Duran, Dubinji, Džalto, Embeli, Emer, Erlik, Fabian, Fabijan, Fai, Fat, Feržan, Grožan, Žanboki, Žamplong, Forir, Foriš, Francia, Francija, Francuski, Francuz, Gabrijel, Gaudenji, Godar, Gubo, Hardi, Hillier, Izan, Kaion, Karaba, Karabaš, Kale, Karleta, Karlo, Karolić, Karolji, Karpti, Leptij, Kler, Kordelie, Kolmar, Kotre, Kudela, Laferton, Laforest, Lahoš, Lampert, Lampret, Lašu, Laubert, Leban, Leleš, Lelik, Leman, Lenard, Lenart, Lenartić, Lenhar, Lenhard, Lenhart, Lener, Lenert, Lerik, Lero, Leto, Letoš, Levang, Lever, Lingli, Loridon, Madon, Mago, Mako, Marg, Margeta, Martan, Marten, Marton, Matiz, Matis, Matiš, Matijas, Mažing, Nišoni, Mofardin, Montanji, Mongejar, Mor, Morar, More, Moric, Mužong, Nater, Obu, Papilion, Parigros, Perdi, Perš, Pete, Petej, Peto, Pomar, Poš, Rago, Reber, Renje, Rifert, Rigo, Roland, Romen, Ronto, Rošival, Sandić, Sebastian, Sebastijan, Bastijančić, Šaronjev, Šaru, Šaberle, Šebeščen, Šebešćan, Šebešćen, Šiket, Šil, Šili, Šilić, Šimon, Šuart, Taš, Taši, Tibold, Tili, Tobijaš, Toma, Tomas, Tomaš, Urs, Uzon, Vabrik, Valentin, Vali, Vavan, Vernot, Volar, Žak, Žaki, Želer, Želi, Žili.

\section{Bibliographie}

ARSENIJEVIĆ 1992 : ARSENIJEVIĆ, Milorad. „Italijanska i zapadnoromanska prezimena u Vojvodini” [Noms de famille italiens et romans occidentaux en Voïvodine]. Thèse de doctorat, soutenue en 1992 à la Faculté de Philosophie de Novi Sad, non-publiée. [orig.] АРСЕНИЈЕВИЋ, Милорад. „Италијанска и западнороманска презимена у Војводини" [Noms de famille italiens et romans occidentaux en Voïvodine]. Необјављена докторска дисертација, одбрањена 1992. године на Филозофском факултету у Новом Саду.

BECKER, 1990 : BECKER, Jean-Marc. « Minorités franciques en Roumanie. L'émigration des Lorrains dans le Banat. » Gewan (Thionville) (1990) : 2-4.

BOTIŞ 1946 : BOTIŞ, Emile. Recherches sur la population française du Banat. Timisoara : L'Association des Descendants d'Anciens Colons Français du Banat (tip. Mathieu).

GOICU-CELAMOF 2007 : GOICU-CELAMOF, Simona. « Les noms de famille des Français installés au Banat. "Nouvlle revue d'onomastique, $\mathrm{n}^{\circ}$ 47-48 (2007) : 115-117. <https:/www.persee.fr/doc/ onoma_0755-7752_2007_num_47_1_1587>

${ }^{17}$ La liste prise de Arsenijević 1992 et complétée par Dipold, Tibold et Šilić. 
GOICU 2000 : GOICU, Simona. « Nume de familie ale francezilor colonizaţi în Banat. » Studii şi cercetări de onomastică, no 5 (2000) : 175-186.

GUDURIĆ 2006 : GUDURIĆ, Snežana. „Neki romanski elementi u srpskom jeziku" [Quelques éléments romans en langue serbe]. Susreti kultura, zbornik radova (2006): 591-598. [orig.] ГУДУРИЋ, Снежана. „Неки романски елементи у српском језику" [Quelques éléments romans en langue serbe], Сусрети култура, зборник радова. (2006): 591-598.

GUDURIĆ 2010 : GUDURIĆ, Snežana. „Toponimi u svetlu jezičkih promena” [Toponymes dans le mirroir des changements linguistiques]. Jezik, književnost, promene. Jezička istraživanja, zbornik radova (2010). Niš: Filozofski fakultet, 397-402. [orig.] ГУДУРИЋ, Снежана. „Топоними у светлу језичких промена [Toponymes dans le mirroir des changements linguistiques]. Језик, књижевност, промене. Језичка истраживања, зборник радова (2010). Ниш : Филозофски факултет, 397-402.

GUDURIĆ 2013 : GUDURIĆ, Snežana. „Jezici i kulture u vremenu na prostoru Srbije. Dva primera iz dva vremena na jednom prostoru." Jezici i kulture u vremenu i prostoru II/1 (2013). Novi Sad: Filozofski fakultet, 157-168. [orig.] ГУДУРИЋ, Снежана. „Језици и културе у времену на простору Србије. Два примера из два времена на једном простору.” Језици и културе у времену и простору II/1 (2013). Нови Сад: Филозофски факултет, 157-168.

GUDURIĆ 2017 : GUDURIĆ, Snežana. „O nekolikim osobenim francuskim tragovima u srpskom. [Les quelques traces particulières françaises en serbe] Ishodišta br. 3 (2017). Temišvar: Savez Srba u Rumuniji, Zapadni Univerzitet iz Temišvara i Filozofski fakultet Univerziteta u Nišu, 87-95. [orig.] ГУДУРИЋ, Снежана. „О неколиким особеним француским траговима у српском " [Les quelques traces particulières françaises en serbe]. Исходишта бр. 3 (2017). Темишвар: Савез Срба у Румунији, Западни Универзитет из Темишвара и Филозофски факултет Универзитета у Нишу, 87-95.

HESS 1928 : HESS, Nicolas. Heimbatbuch der drei Schwestergemeinden Sveti-Hubert, Charlevil und Soltur im Banat 1770-1927. Sv.-Hubert/ Veliki Betschkerek.

MILLEKER 1937 : MILLEKER, Felix. Versuch einer Einsiedelung von Spanien im Banat. Wrschatz.

PETROV 1974 : PETROV, Milan. Svilarstvo u Vojvodstvu Srbija i Tamiški Banat 1850-1860 [Élevage du ver à soir en Voïvodstvo Serbie et Banat Tamichois]. Novi Sad: Institut za izučavanje istorije Vojvodine / Prosveta. [orig.] ПЕТРОВ, Милан. Свиларство у Војводству Србија и Тамишки Банат 1850-1860. [Élevage du ver à soir en Voïvodstvo Serbie et Banat Tamichois] Нови Сад: Институт за изучавање историје Војводине / Просвета. 
POPOVIĆ 1959 : POPOVIĆ, Dušan. Srbi u Vojvodini [Les Serbes en Voïvodine] I-III. Novi Sad: Matica srpska. [orig.] ПОПОВИЋ, Душан. Срби у Војводини [Les Serbes en Voïvodine] I-III. Нови Сад: Матица српска.

ROSAMBERT 1952 : ROSAMBERT, André. Émigrations et implantation occidentales en Europe sudorientale. Nancy : Centre Européen Universsitaire.

STANGLICA 1935: STANGLICA, Franz. Die Auswanderung der Lothringer in das Banat und die Batschka im 18 Jahrhundert. Frankfurt a.M.

\section{Снежана У. Гудурић}

\section{У ПОТРАЗИ ЗА ФРАНЦУЗИМА У ВОЈВОДИНИ}

Војводина, аутономна покрајина на северу Републике Србије, увек је представљала својеврстан европски мозаик народа, језика и култура. Нас у овом тексту занима присуство Француза на територији Војводине, будући да присуство њиховог језика и културе више није видљиво на овим просторима. Током XX века, аустроугарска монархија плански је спровела три колонизације, током којих се знатан број Француза доселио у јужну Угарску, данашњу Војводину, пре свега у Банат. За разлику од припадника неких других народа који су успели да сачувају свој идентитет негујући свој језик и културу током више од два века, Французи су се релативно брзо асимиловали у суседне етницитете. Па ипак, брижљиво историјскојезичко истраживање открива код појединих војвођанских презимена, као и код неколицине банатских микротопонима, скривене француске трагове. Првобитни облици презимена и микротопонима претрпели су током времена знатне фонетско-фонолошке промене, највише под утицајем германизације, мађаризације и србизације, али паралелна анализа одговарајуће историјске и језичке грађе омогућила је да се ти првобитни облици установе и попишу како не би остали изгубљени у времену.

У овом раду представљају се резултати тих историјских и језичких истраживања, допуњени новим сазнањима, који недвосмислено упућују на француске корене појединих наших сународника. Највећу захвалност што се ти корени нису потпуно изгубили кроз векове дугујемо Николасу Хесу, Срећку Милекеру, Францу Штанглици, Андреу Розамберу и понајвише Милораду Арсенијевићу који је у својој докторској дисертацији објединио претходна истраживања и употпунио их својим, спроведеним током последњих деценија претходног века.

Кључне речи: ономастика, Војводина, Банат, колонизација, Французи, Милорад Арсенијевић 